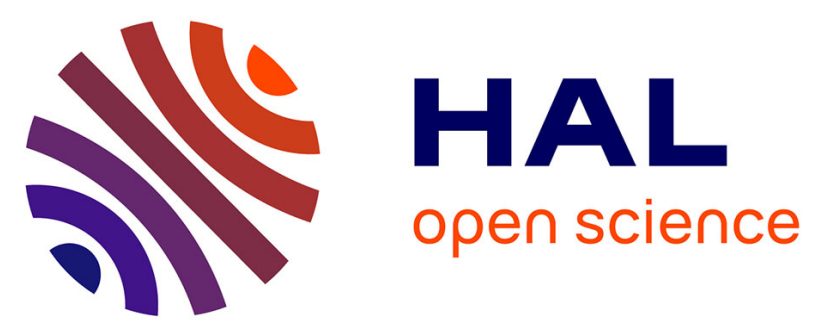

\title{
Advances in X-ray diffraction contrast tomography: flexibility in the setup geometry and application to multiphase materials
}

Péter Reischig, Andrew King, Laura Nervo, Nicola Viganò, Yoann Guilhem, Willem Jan Palenstijn, K. Joost Batenburg, Michael Preuss, Wolfgang Ludwig

\section{To cite this version:}

Péter Reischig, Andrew King, Laura Nervo, Nicola Viganò, Yoann Guilhem, et al.. Advances in X-ray diffraction contrast tomography: flexibility in the setup geometry and application to multiphase materials. Journal of Applied Crystallography, 2013, 46 (2), pp.297-311. 10.1107/S0021889813002604 . hal-01553410

\author{
HAL Id: hal-01553410 \\ https://hal.science/hal-01553410
}

Submitted on 3 Jul 2017

HAL is a multi-disciplinary open access archive for the deposit and dissemination of scientific research documents, whether they are published or not. The documents may come from teaching and research institutions in France or abroad, or from public or private research centers.
L'archive ouverte pluridisciplinaire HAL, est destinée au dépôt et à la diffusion de documents scientifiques de niveau recherche, publiés ou non, émanant des établissements d'enseignement et de recherche français ou étrangers, des laboratoires publics ou privés. 
Journal of

\section{Applied Crystallography}

ISSN 0021-8898

Received 8 October 2012

Accepted 25 January 2013
(C) 2013 International Union of Crystallography Printed in Singapore - all rights reserved

\section{Advances in X-ray diffraction contrast tomography: flexibility in the setup geometry and application to multiphase materials ${ }^{1}$}

\author{
Péter Reischig, ${ }^{\text {a,b,c } * *}$ Andrew King, ${ }^{c}$ Laura Nervo, ${ }^{c, d}$ Nicola Viganó, ${ }^{b, c}$ Yoann \\ Guilhem, ${ }^{\mathrm{b}, \mathrm{c}}$ Willem Jan Palenstijn, ${ }^{\mathrm{e}}$ K. Joost Batenburg, ${ }^{\mathrm{e}, \mathrm{f}}$ Michael Preuss ${ }^{\mathrm{d}}$ and \\ Wolfgang Ludwig ${ }^{\mathrm{b}, \mathrm{c}}$
}

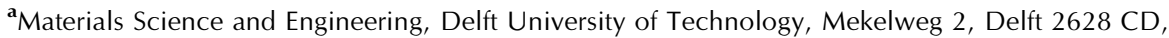
The Netherlands, ${ }^{\mathbf{b}}$ MATEIS, INSA-Lyon, 7 Avenue Jean Capelle, Villeurbanne 69621, France, ${ }^{{ }^{2} E S R F,} 6$ rue Jules Horowitz, Grenoble 38043, France, ${ }^{\mathbf{d}}$ School of Materials, Room E29, Materials Science Building, University of Manchester, Manchester M13 9PL, UK, ${ }^{\mathbf{e} V i s i o n ~ L a b, ~ B u i l d i n g ~ N, ~}$ Universiteitsplein 1, University of Antwerp (CDE), Wilrijk (Antwerp) 2610, Belgium, and ${ }^{\mathbf{f}}$ Scientific Computing, Centrum Wiskunde \& Informatica, Science Park 123, Amsterdam 1098 XG, The Netherlands. Correspondence e-mail: wolfgang.ludwig@esrf.fr
\end{abstract}

Diffraction contrast tomography is a near-field diffraction-based imaging technique that provides high-resolution grain maps of polycrystalline materials simultaneously with the orientation and average elastic strain tensor components of the individual grains with an accuracy of a few times $10^{-4}$. Recent improvements that have been introduced into the data analysis are described. The ability to process data from arbitrary detector positions allows for optimization of the experimental setup for higher spatial or strain resolution, including high Bragg angles $\left(0<2 \theta<180^{\circ}\right)$. The geometry refinement, grain indexing and strain analysis are based on Friedel pairs of diffraction spots and can handle thousands of grains in single- or multiphase materials. The grain reconstruction is performed with a simultaneous iterative reconstruction technique using three-dimensional oblique angle projections and GPU acceleration. The improvements are demonstrated with the following experimental examples: (1) uranium oxide mapped at high spatial resolution $(300 \mathrm{~nm}$ voxel size); (2) combined grain mapping and section topography at high Bragg angles of an Al-Li alloy; (3) ferrite and austenite crystals in a dual-phase steel; (4) grain mapping and elastic strains of a commercially pure titanium sample containing 1755 grains.

\section{Introduction}

X-ray diffraction contrast tomography (DCT) is a nondestructive characterization method providing access to the three-dimensional grain microstructures in a wide range of polycrystalline materials (King et al., 2008; Syha et al., 2012; Rolland du Roscoat et al., 2011). The technique combines the concepts of image reconstruction from projections (tomography) and X-ray diffraction imaging (topography) and complements the portfolio of the synchrotron-based threedimensional X-ray diffraction techniques (3DXRD) described by Poulsen (2012). The only complete data processing software using the DCT approach published so far has been developed in the 'Graintracking' project by a collaboration of research teams at the National Centre for Scientific Research (CNRS, France), the European Synchrotron Radiation Facility (ESRF, Grenoble, France) and the University of Manchester (UK).

\footnotetext{
1 This article forms part of a special issue dedicated to advanced diffraction imaging methods of materials, which will be published as a virtual special issue of the journal in 2013.
}

\subsection{Combined three-dimensional microstructural informa- tion}

DCT is a truly three-dimensional tomographic imaging approach, sharing a common experimental setup with conventional X-ray microtomography. After interaction with the material, both the transmitted and diffracted beams are recorded on a high-resolution X-ray imaging detector positioned close to the sample. The three-dimensional distribution of the X-ray attenuation coefficient, and the three-dimensional shape, grain average orientation and elastic strain tensor of all grains in the illuminated sample volume, are determined from analysis of the transmitted and diffracted intensities, respectively. The spatial accuracy of the absorption reconstruction is comparable to the pixel size of the detector and is a few pixels for the grain boundaries (Ludwig et al., 2008). The angular sensitivity is of the order of $0.05^{\circ}$ and the precision of the elastic strain components is at the level of a few times $10^{-4}$ (Reischig, 2008). The accuracies are strongly dependent on the experimental conditions and the sample itself. 
Table 1

Practical limits and typically used values of the acquisition parameters in diffraction contrast tomography.

\begin{tabular}{lll}
\hline Parameter & Practical limits & Typical range \\
\hline Effective pixel size $(\mu \mathrm{m})$ & $0.3-50$ & $1-10$ \\
Grain size $(\mu \mathrm{m})$ & $10-5000$ & $20-200$ \\
Sample diameter $(\mathrm{mm})$ & $0.05-10$ & $0.3-1.5$ \\
Rotation axis-detector distance $(\mathrm{mm})$ & $0.5-100$ & $3-10$ \\
No. of pixels in sensor & $1024 \times 1024$ to & $2048 \times 2048$ \\
& $4096 \times 4096$ & \\
Rotational increment $\left(^{\circ}\right)$ & $0.01-1$ & $0.05-0.5$ \\
Width of $2 \theta$ range $\left(^{\circ}\right)$ & $5-45$ & $10-30$ \\
Accessible $2 \theta$ range $\left(^{\circ}\right)$ & $0-180$ & $10-30$ \\
Accessible energy range $(\mathrm{keV})$ & $6-100$ & $15-50$ \\
\hline
\end{tabular}

Given an X-ray beam with a sufficient degree of partial coherence, the acquisition of an additional tomographic scan at larger sample-to-detector distance and optimized image resolution adds another complementary X-ray imaging mode: propagation-based X-ray phase contrast imaging (PCT) (Cloetens et al., 1997). PCT is sensitive to weak differences in electron density and can reveal microstructural features that cannot be resolved in the absorption contrast image.

The combination of multiple nondestructive three-dimensional characterization modes on the same instrument offers unique possibilities for studying the time evolution of damage and deformation processes in structural materials. Very much like electron backscatter diffraction (EBSD) has enhanced the possibilities of conventional scanning electron microscopy by adding complementary crystallographic information, DCT enhances the possibilities of three-dimensional X-ray imaging, providing insight into hitherto inaccessible aspects of microstructure, and their evolution as a function of time, in bulk materials (Herbig et al., 2011; Ludwig, King et al., 2009; King et al., 2011).

Related techniques of the 3DXRD type which can also deliver subgrain information use forward simulation and raytracing approaches (Alpers et al., 2006; Suter et al., 2006; Schmidt et al., 2008) to reconstruct orientation maps from experimental data of sample cross sections. These data are acquired with a focused line beam, which reduces the complexity of the underlying reconstruction task. In this case, three-dimensional sample volumes are obtained by stacking multiple two-dimensional measurements. Other methods using a polychromatic X-ray beam and Laue microdiffraction can provide three-dimensional orientation and strain information at the subgrain level (Larson et al., 2002; Tamura et al., 2002), but the long scanning times significantly limit the size of the measured region.

\subsection{Experimental setup and principles}

The specifications required for the DCT technique are attainable on a microtomograph or a dedicated diffraction instrument; thus synchrotron beamlines specialized for imaging and diffraction can generally be considered for the experiments.
The sample is placed on a rotation stage and irradiated by a parallel monochromatic synchrotron $\mathrm{X}$-ray beam that is perpendicular to the rotation axis and whose dimensions are defined by slits (Fig. 1). As the polycrystalline sample is rotated during the scan, the Bragg condition for diffraction is fulfilled at specific angular positions for the various atomic lattice planes in the grains. At those positions where diffraction occurs, some of the incoming intensity is diverted out of the direct beam. The direct beam and the diffracted beams from the specimen are recorded on a high-resolution (0.3$20 \mu \mathrm{m}$ effective pixel size) and high dynamic range imaging detector (Labiche et al., 2007) placed within a close distance (order of $1-10 \mathrm{~mm}$ ). The images are integrated over small angular increments $\left(0.05-0.5^{\circ}\right)$ covering $360^{\circ}$. In the data processing, diffraction spots are segmented and indexed according to their grain of origin to then be used as projections for the grain shape reconstruction. While earlier DCT variants (Ludwig et al., 2008; Johnson et al., 2008) also used the extinction contrast in the direct beam, the current processing is based entirely on diffraction spots.

Commonly used imaging sensors have typically $2048 \times 2048$ pixels, and using appropriate optical systems, the effective pixel size $p$ can typically be adjusted to between 0.3 and $20 \mu \mathrm{m}$. The actual pixel size and sample size in a DCT experiment is chosen as a function of the average grain size $d$ of the material such that the $d / p$ ratio is somewhere between 20 and 100 and that the sample dimensions in the direction perpendicular to the rotation axis do not exceed 600 pixels. Table 1 summarizes the practical limits, and the values routinely used, for the most important DCT acquisition parameters.

\section{Advances in the DCT data processing}

The principal logic of the processing route has not changed compared to what is described by Ludwig et al. (2008). Friedel pairs are used as a basis for finding grain positions and

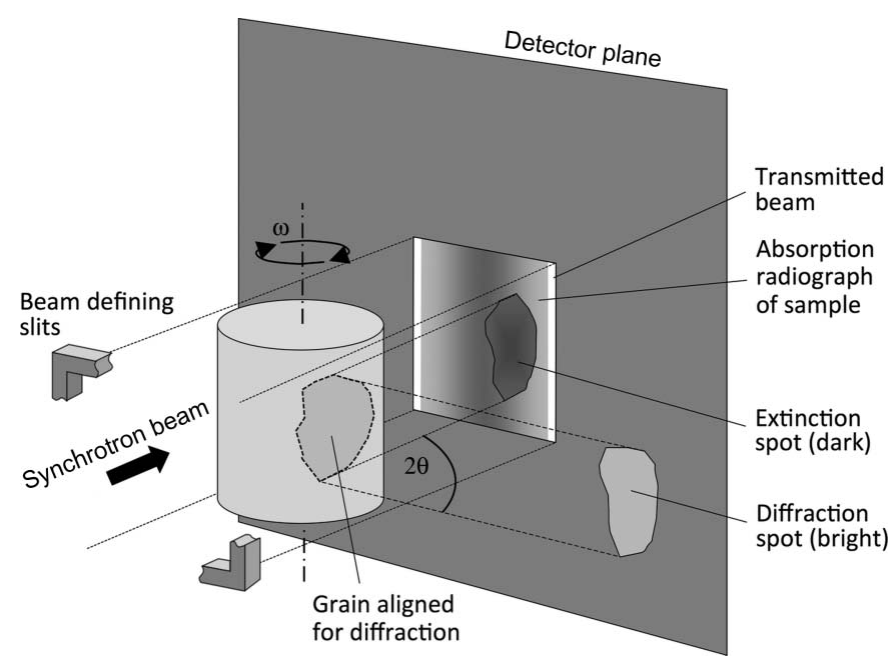

Figure 1

Experimental setup for diffraction contrast tomography, showing both the incident and the diffracting beam from a grain impinging on the same near-field detector. 
orientations by indexing diffraction spots that were segmented from the preprocessed image stack. The grains are then reconstructed individually in three dimensions and assembled to create a space-filling grain map under a mask found from the absorption reconstruction.

The DCT processing route consists of the following main steps:

(1) Preprocessing of the image stack

(2) Segmentation of diffraction spots

(3) Matching of Friedel pairs

(4) Indexing of grains from Friedel pairs; alternatively fitting strain tensors

(5) Selection of diffraction spots for grain reconstruction

(6) Reconstruction of grain shapes

(7) Assembly of the grain map

Steps 1, 2 and 7 are essentially the same as described in earlier publications. In this article, a summary of the main processing stages with an emphasis on the more recent improvements is presented. A fitting procedure to compute the complete average elastic strain tensor from such near-field data sets is also briefly outlined and illustrated.

Processing of 3DXRD or DCT type experimental data still requires a significant amount of monitoring and user interaction owing to the vast variety of samples and experimental conditions. The interactive stages of the DCT processing route have been greatly improved by reduced processing times and the addition of graphical user interfaces. These softwarerelated features will be discussed elsewhere.

\subsection{Preprocessing and segmentation}

The preprocessing step involves image-processing steps on the entire image stack. A distortion correction is performed using a distortion map based on the radiograph of a regular absorption grid. The footprint of the direct beam and boundaries of the diffracted beam area in the images are determined. Constant background features are removed from the diffraction area by a moving median filter through the image stack. The direct beam radiographs are used for an absorption reconstruction through either a conventional filtered backprojection or a three-dimensional simultaneous iterative reconstruction technique (SIRT) algorithm from the ASTRA library (Palenstijn et al., 2012, 2011).

The segmentation involves finding diffraction signals in the diffracted beam area. This can be done in two different ways: (1) a single threshold at the background noise level - areas above this threshold are segmented as diffraction spots; (2) double threshold segmentation - possible peaks are identified with an intensity threshold and a second threshold that is linearly proportional to the given peak intensity is used for determining the outlines of the spots. Each diffraction spot, also called a diffraction blob hereafter, is segmented as a connected neighbourhood using a three-dimensional morphological reconstruction (region growing). Overlapping spots may be segmented as one and are filtered out in later stages. All diffraction metadata are stored in a database.

Instead of simple thresholding, 'soft' thresholding has been recently applied to improve the grain shape reconstructions: that is, the threshold that determined the background noise level or the minimum value in a blob is subtracted from the entire blob volume. Thus, the intensity approaches zero at the edges of the blobs, which results in smoother and physically more consistent grain shapes.

\subsection{Arbitrary setup geometry}

A general geometric description has been developed to allow for indexing and reconstruction of any near-field or farfield 3DXRD type experimental data that suit the general prerequisites of the DCT method: the number of grains and/or the mosaicity (plastic deformation) of the grains is limited to avoid excessive overlap and distortion of the diffraction spots.

The setup is defined in the $(x, y, z)$ laboratory reference frame, which is a right-handed Cartesian coordinate system. This laboratory reference frame is not fixed to anything, but the position and direction of the setup components are freely defined with respect to it. Therefore the setup can be defined arbitrarily at the user's convenience; it is only the relative position of the components that eventually matters.

The position and orientation of the incoming beam, rotation axis and detector are described with their laboratory coordinates in Fig. 2. Initially, approximate values have to be specified by the user and a refinement of these global parameters can be done later by fitting (see \$2.4).

The detector reference frame is a two-dimensional planar coordinate system defined relative to the laboratory reference frame by $\mathbf{u}$ and $\mathbf{v}$ unit vectors that are not necessarily perpendicular to each other. It represents the images obtained after distortion correction. Depending on how the distortion correction is carried out, it may leave a residual shear and stretch in the images. The free choice of $\mathbf{u}, \mathbf{v}$ and their corresponding pixel sizes offers the possibility to take those residual distortion effects into account, and can accommodate

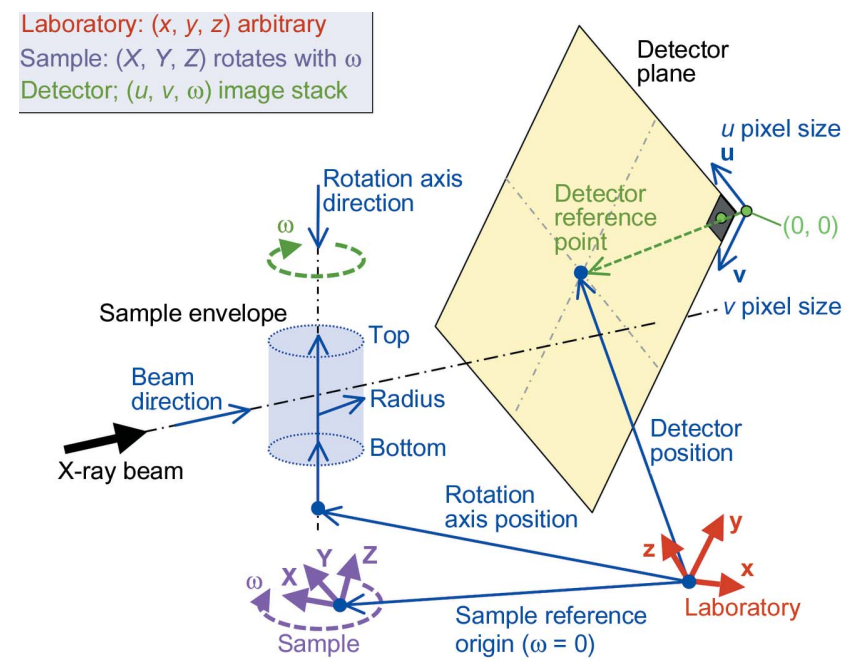

Figure 2

The arbitrary DCT experimental geometry and its three reference frames. The parameters defining the relative position of the incident beam, sample envelope and detector position in the laboratory reference frame are shown. 
any flips or rotations of the raw images arising from the detector system.

The sample reference frame $(X, Y, Z)$ describes a regular grid on which grain positions and orientations are calculated in the Friedel pair matching and indexing phases. It is fixed to the rotation stage and rotates together with the specimen. The rotational position is described by the $\omega$ angle. The origin and base vectors of the sample reference frame are also defined relative to the laboratory reference frame at $\omega=0$. It is not necessarily a Cartesian coordinate system and the voxel sizes may be different along each axis.

The reconstruction reference frame defines the grid on which the voxellated grain map is reconstructed. It is like the sample reference frame but it may have different grid spacings and orientation.

\subsection{Friedel pair matching at high Bragg angles and in multiphase materials}

Friedel pairs are $h k l$ and $\overline{h k l}$ reflections from the same grain that occur at $180^{\circ}$ offset in the scan if the rotation axis is perpendicular to the beam. They are found by applying crystallographic and spatial search criteria along with the metadata of the diffraction spots (Ludwig, Reischig et al., 2009). Friedel pairs provide relatively high orientation accuracy in near-field data sets before the indexing stage by eliminating the uncertainty in the grain positions. The search algorithm has been updated to benefit from the arbitrary geometry framework and now allows Friedel pairs to be found for any suitable detector position up to $180^{\circ} 2 \theta$. This enables the use of detectors perpendicular to the beam or in backscattering mode, even outside the path of the direct beam. Friedel pairs can be observed when the centre of the detector and the sample lie close to the same plane perpendicular to the rotation axis.

To understand the pair matching geometry, one can imagine a virtual setup in the sample reference frame where the beam and detector are rotated around a fixed sample (Fig. 3a). A grain that diffracts into point $\mathrm{A}$ on the detector at rotation angle $\omega$ will also be in the Bragg condition for diffraction and will give rise to diffraction spot $\mathrm{B}$ at $\omega+180^{\circ}$. Simple alignment procedures can ensure that the rotation axis is perpendicular to the beam with a precision of better than $0.01^{\circ}$. Any crystallographic plane in a grain can give rise to a maximum of four reflections, that is two Friedel pairs, during a $360^{\circ}$ rotation. The relative positions of the four spots on the detector are shown in Fig. 3(b).

For any Friedel pair the diffracted beam path is obtained as the straight line connecting A and B (see Fig. $3 a$ ). The grain of origin must lie along this line but there is no need to determine the actual grain position at this stage. The direction of the scattering vector can be calculated as the vector difference between the diffracted and incident beams. The Bragg angle $\theta$ and azimuthal angle $\eta$ are also calculated, as well as the difference from the nearest theoretical $\theta$ value, average spot size and intensity for the pair.
The search is restricted by the spatial constraint that the diffracted beam path has to cross the sample envelope - a cylindrical gauge volume along the rotation axis irradiated by the beam. In practice, this is realized by only considering pairs inside the bounding box of the sample envelope projected onto the opposite detector from point A (Fig. 3). The sample envelope is determined from the direct beam footprint, if available, or estimated according to the beam size.

Pair combinations that pass the preset tolerances for deviations in $\theta$, mean $\omega$ value, beginning and end of the $\omega$ range, bounding box size, intensity, and area are considered in the search. A figure of merit to describe the mean error of a pair in a single value is calculated as the summed square of those deviations weighted by the squared inverse of its corresponding tolerance limit. The final set of Friedel pairs is selected from all potential pair combinations ranked according to their mean error and includes any diffraction spot maximum once.

Higher Bragg angles (higher Miller indices, smaller lattice spacings) imply that many more reflections fall in the same $\theta$ range and different $\{h k l\}$ reflections may have close or even identical $\theta$ angles. This prevents the unambiguous assignment of the $\{h k l\}$ family to the Friedel pairs. Multiphase materials constitute the same challenge, as a given $\theta$ angle may belong to more than one phase. The pair matching method is visualized on a dual-phase steel sample in Fig. 4. Intragranular strain may accentuate the ambiguity between the phases owing to a wider $\theta$ distribution for a given $\{h k l\}$ family. The problem has been overcome by assigning more than one possible phase and $\{h k l\}$ family to a Friedel pair inside the acceptance ranges around

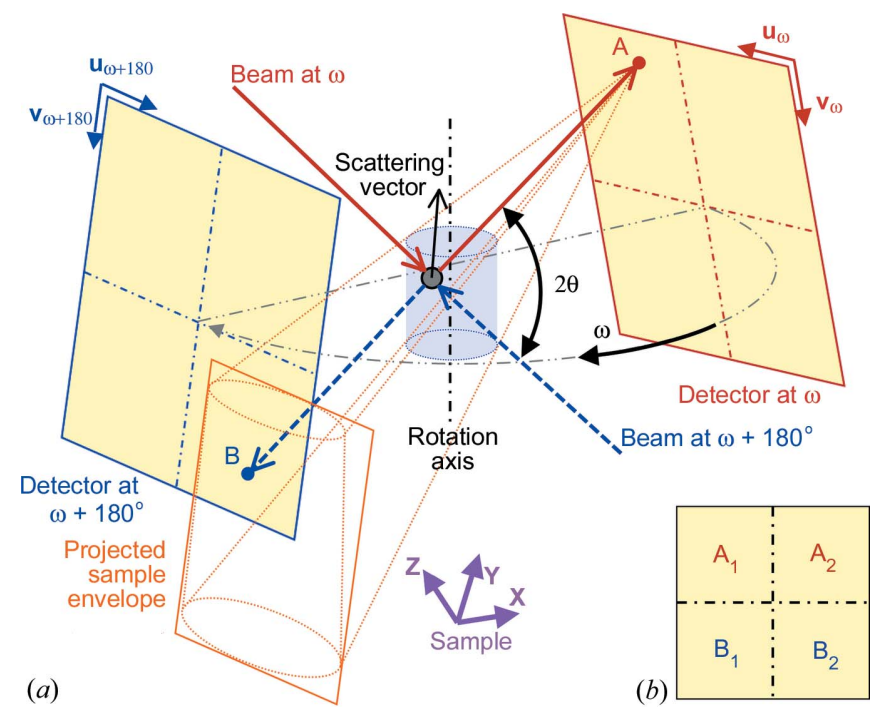

Figure 3

Geometry of Friedel pairs in an arbitrary detector position. A Friedel pair A-B is illustrated in the reference frame fixed to the sample. The incident beam and the detector are shown $180^{\circ}$ offset in $\omega$. The two diffracted beam paths are coincident and connect A with B through the grain. The diffraction angles can be calculated directly without precise knowledge about the grain position. B falls inside the projection of the sample envelope from A onto the detector in the opposite position. (b) Relative positions of the four diffraction spots from an $(h k l)$ crystallographic plane of a grain on the detector. The two Friedel pairs are $A_{1}-B_{1}$ and $A_{2}-B_{2}$. 
the theoretical $\theta$ values. All potential $\{h k l\}$ and phase assignments of a Friedel pair are considered in the indexing routine.

Usually only about half of the diffraction spots are paired owing to one of the pair of spots not falling on the detector or falling in the direct beam area, faulty segmentation, or spot overlaps. It is acceptable to include wrong matches in the processing up to a few percent as these yield a random scattering vector and most probably will not be indexed as pairs but can be indexed later as individual spots (see $\$ 2.5$ ).

Friedel pairs can be exploited in far-field data too and have been used for indexing (Moscicki et al., 2009), strain measurements (Aydiner et al., 2009) and setup calibration (Sharma et al., 2012a).

\subsection{Refinement of the arbitrary setup geometry}

Calibration of the exact detector position relative to the beam and the rotation axis is essential to achieve high orientation and three-dimensional shape reconstruction accuracy. While the Debye-Sherrer rings from a powder sample of a standard material can be used to calibrate a far-field detector, this procedure is not suitable for a near-field setup. The method of using Friedel pairs for the geometry refinement described by Reischig (2008) has been adapted to calibrate arbitrary detector positions or the crystal lattice parameters. A

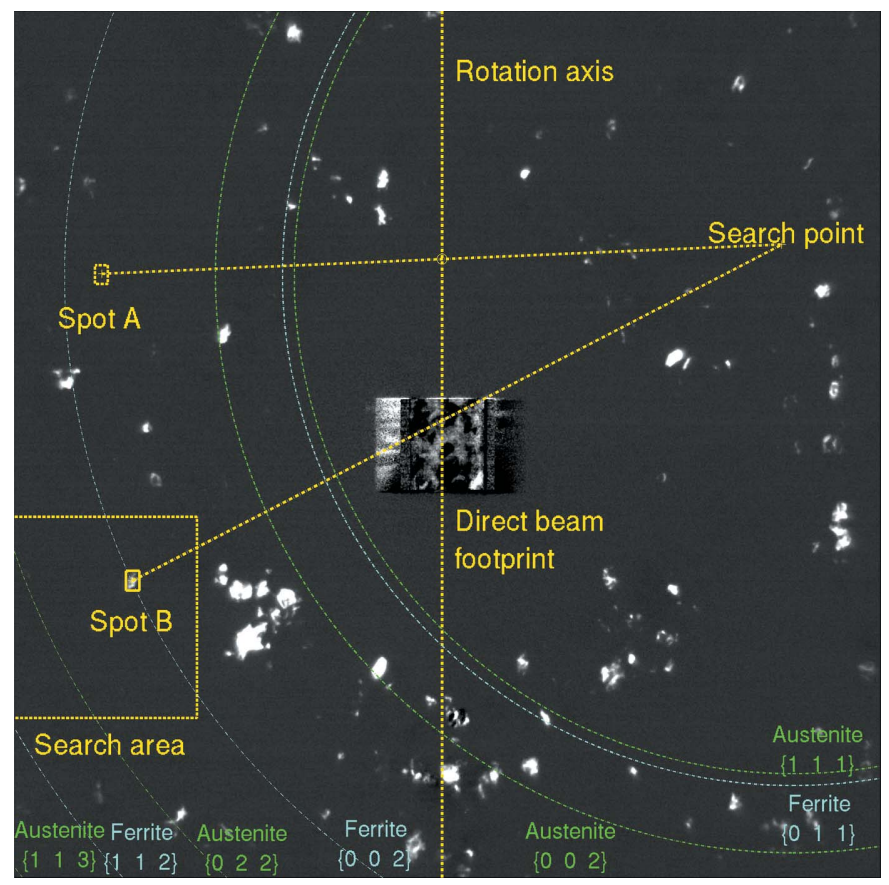

Figure 4

A preprocessed image from an inline detector showing the search for Friedel pairs from a duplex steel sample. Diffraction spot A was recorded in another image $180^{\circ}$ offset in $\omega$. Following a $180^{\circ}$ rotation around the axis, a search point shows its position in the sample reference frame relative to the displayed image. From this search point, the Bragg angle for Friedel pair B can be calculated. Lines (conic sections) show the positions in the image that correspond to the Bragg angles of the $\{h k l\}$ reflections from the ferrite and austenite phases. The search is restricted to spots that result in a valid Bragg angle and are inside the sample envelope projected from the search point onto the detector plane. somewhat different approach based on Friedel pairs is described by Sharma et al. (2012b). Other calibration methods for 3DXRD are reported by Bernier et al. (2011) and Oddershede et al. (2010). A technique for calibrating the setup parameters of a diffractometer equipped with an area detector using a single crystal centred on the goniometer is described by Paciorek et al. (1999).

As the diffraction angles can be computed from the pairs without knowing the grain centroids, the refinement can be performed before the indexing stage. This refinement is applicable with a single-crystal or polycrystal standard that exhibits little or no residual stresses, or can be performed using the actual specimen if its lattice parameters are known.

The $\{h k l\}$ family and the corresponding theoretical $d$ spacing yield an X-ray wavelength for any Friedel pair via Bragg's law. A nonlinear least-squares optimization algorithm is applied to minimize a target function - the sum of the squared deviation of the computed X-ray wavelengths from the true value. This implies that the beam energy should be carefully characterized during the experiment, either using an absorption edge or a standard Si crystal (Bond method). The detector position is refined in the laboratory reference frame, which at the same time defines its position relative to the beam and rotation axis, since these are kept constant and usually are fixed to the laboratory base. Instead of the parameters shown in Fig. 2, however, the following more intuitive but equivalent parameters can be fitted:

(a) tilt around the $u$ axis

(b) tilt around the $v$ axis

(c) in-plane tilt

(d) angle between the $u$ and $v$ axes

(e) mean $u$ and $v$ pixel size

(f) pixel size ratio $u / v$

( $g)(x, y, z)$ position of the detector (distance and offset)

Any subset of those parameters selected by the user can be fitted simultaneously and the original base parameters are adjusted accordingly. The tilts are applied as a sequential rotation around the initial $u$ and $v$ axes and the detector plane normal.

Refinement of the $u-v$ angle and pixel sizes may be desirable for detector systems with a scintillator and visible light optics, if shear and stretches in the image have not been eliminated during the distortion correction (Reischig, 2008).

From the diffraction geometry, only either the mean pixel size or the rotation axis-detector distance can be fitted at any one time, as they have the inverse effect. The mean pixel size of the detector system is generally known or measured, and the distance is fitted since it is especially difficult to measure precisely for a near-field detector.

The detector position in the direction parallel to the rotation axis is a free parameter and cannot be determined from Friedel pairs, as the scattering geometry is unaffected by such a displacement (see Fig. 3). It only matters when data are merged from multiple detectors and their relative position has to be adequately set.

If the geometry is known or has been previously fitted, the crystal lattice parameters for any phase can be fitted with the 
same optimization procedure. In the case of multiphase materials, the basis of the fitting can be chosen as the phase for which the lattice parameters are known with the smallest uncertainty. It is reasonable to choose a phase with an exact stoichiometric composition or one with a narrow solubility region for alloying elements. Another aspect to be considered is that the number of free lattice parameters should be small (e.g. one for a cubic lattice). The geometry and the lattice parameters of the other phase can then be calibrated according to that basis to result in a consistent measurement.

\subsection{Indexing}

Indexing is the process of finding grain positions and orientations in the sample by grouping the observed diffraction spots. It is practically the same problem as in the far-field case. Far-field data have better orientation accuracy, while near-field data contain more precise spatial and size information about the grains. The effectiveness of the indexing depends on the extent and accuracy of the information on which it primarily relies. While the first indexing algorithms relied on orientation and intensity (Lauridsen et al., 2001), more recent ones also include spatial information and, sometimes, iteration steps that contribute to the reliability and robustness of the indexing, at the expense of computational complexity and time (Ludwig, Reischig et al., 2009; Moscicki et al., 2009; Lyckegaard et al., 2010; Bernier et al., 2011; Sharma et al., 2012a,b; ImageD11, http://sourceforge.net/apps/trac/fable/ wiki/imaged11; GrainSpotter, http://sourceforge.net/apps/trac/ fable/wiki/grainspotter).

An updated version of the iterative indexing algorithm Indexter (Ludwig, Reischig et al., 2009) is employed to find grains using all the available information from each Friedel pair. The parameters measured from a pair are the following: potential $\{h k l\}$ families and phases, scattering vector direction, diffracted beam path through the sample volume, average bounding box sizes, intensity, and area of the two diffraction spots. Improvements to the earlier published version include a faster orientation search in Rodrigues space, the ability to account for multiphase materials, the posterior addition of single spots to the grains, and the possibility to detect and remove overlapping diffraction spots from the grains (steps 5 and 6 in Fig. 5).

Each phase in a multiphase material is indexed independently, by considering only those Friedel pairs for which the Bragg angle was found to be close enough to one of the given phases in the pair matching stage.

The core of the algorithm is an iterative search in which the tolerances for finding grains are gradually loosened. This ensures robustness as erroneously segmented diffraction spots or wrong Friedel pair matches, as well as coincident 'fake' grains in a large data set containing many spots, are less likely to be indexed. The first iteration loops are expected to index Friedel pairs with the smallest angular and positional error, and the last loops can account for higher errors but in a much reduced set of pairs. The preset tolerance values can be revised and adapted to the actual data set by the user for each indexing stage by setting values for the first and last iteration loops. Although this means a higher number of parameters to be chosen, finding an appropriate set of parameters for a new sample material tends to be relatively easy in a few trial runs. Thanks to the iterative approach and the subsequent statistical refinement, very similar results are usually obtained with a conveniently wide range of parameters.

Grain orientations are handled in Rodrigues space as described by Poulsen (2004). A three-dimensional Rodrigues space provides a mapping of the orientation space where each point uniquely defines a three-dimensional rotation. Crystal symmetry is accounted for by restricting this space to the fundamental zone, that is, a polyhedron dependent on the rotational symmetries of the actual lattice system or space group. The orientation of a grain is described as the smallest rotation around any axis from a reference orientation fixed to the sample reference frame. Triclinic or monoclinic crystals cannot be directly handled this way, as the Rodrigues space in these cases would be infinite in size. Each potential $h k l$ and $\overline{h k l}$ reflection assigned to a Friedel pair of a given phase represents

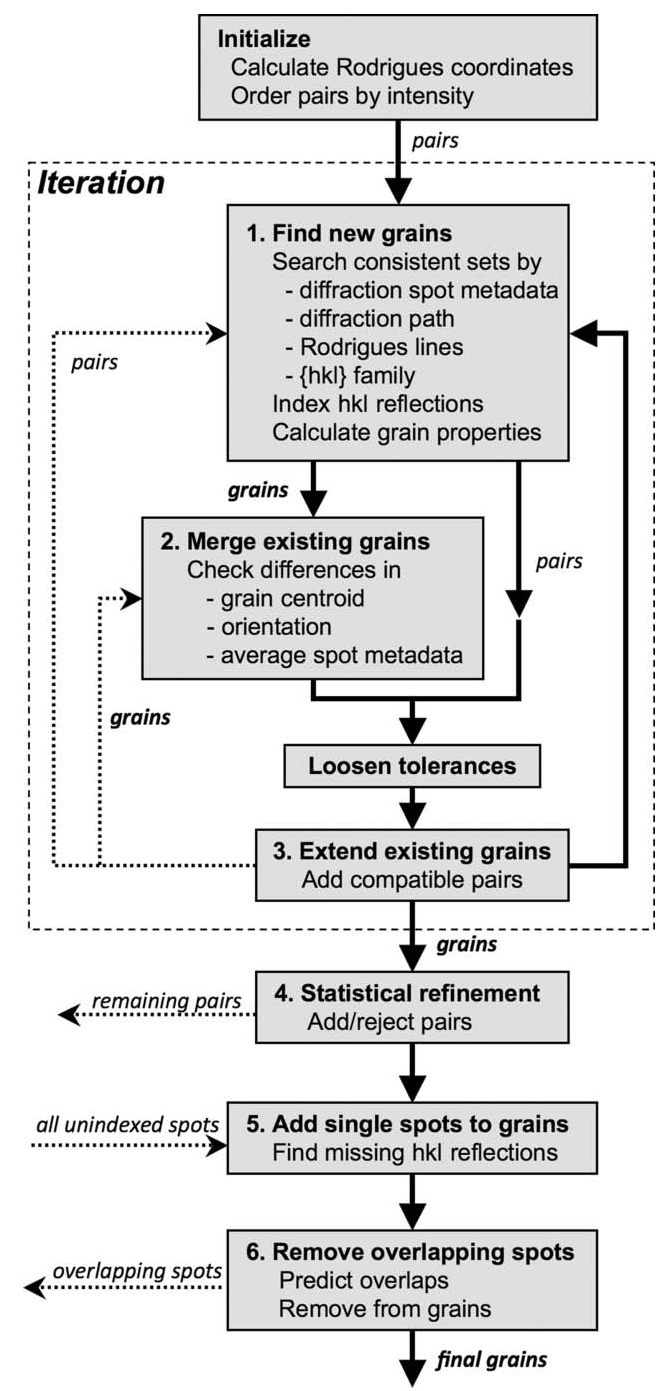

Figure 5

Flow-chart of the indexing algorithm that is applied to each crystal phase present in the sample. 
a straight line through Rodrigues space that crosses the fundamental zone. Line segments outside the fundamental zone correspond to symmetry equivalents and are folded back into this zone, resulting in a set of $m$ line segments, where $m$ is the multiplicity of the $h k l$ reflection. An extended fundamental zone is also defined and applied in parallel with the exact one to account for measurement errors when searching for the intersection point of line segments corresponding to different reflections of the same grain.

In the initialization step, the Rodrigues search lines for all pairs are pre-calculated and stored in memory to save computation time later. Compatible angles between all combinations of two $(h k l)$ plane normals are also precomputed and stored in a lookup table. The Friedel pairs are ordered according to their average spot intensity to speed up searches in stages 1 and 3 , as pairs with similar intensities are more likely to belong to the same grain.

Stages 1-3 of the process are applied iteratively, while stages $4-6$ are sequential refinements:

(1) Finding grains: new grains are found by a consistent search for small subsets of pairs that may constitute a grain. A subset must contain a preset minimum (usually 3-5) of Friedel pairs which are all consistent pairwise. Consistency is checked by comparing bounding box sizes, intensity, area and the angle between the scattering vectors (plane normals). Furthermore, their diffracted beam paths need to intersect at the same point (within errors) in the sample envelope, as well as their Rodrigues lines in the (extended) fundamental zone. In order to optimize speed, consistency criteria are applied in an increasing order of approximate computation time - the latter two criteria being the most demanding - and only in the case of positive results from the previous checks. Grain locations, orientations and the specific $\{h k l\}$ family in the grain are assigned to the indexed Friedel pairs.

(2) Merging grains: Friedel pairs that originate from the same grain are often erroneously split between two or more grains as they only fulfil the constraints as separate subsets in stage 1 in the early iteration steps. These grains are identified as having close centroid location, orientation and spot properties, and are merged into one grain.

(3) Extending grains: the previous tolerances used in stage 1 are loosened to let more Friedel pairs be assigned to existing grains.

(4) Statistical refinement: statistics on the deviation of the plane normals and diffracted beam paths from the average expected values and on the spot properties are calculated for every grain. Additional pairs whose properties fall closer than a few (usually $3-4$ ) standard deviations from the mean values are included in the grain. Outlier pairs are identified and excluded in a similar manner. The strength of this additional refinement is that it depends little on the parameters preset by the user but primarily on the actual data set.

(5) Adding single spots: once the grains have been indexed, a scattering vector and $h k l$ reflection for any diffraction spot can be determined with respect to the centre of a grain. Unpaired and paired but unindexed diffraction spots can thus be tested for consistency on the basis of the statistical prin- ciples mentioned above and can be included in the indexed grains.

(6) Removal of overlapping spots: the expected diffraction spot positions for all grains and all $h \mathrm{kl}$ reflections are calculated to account for diffraction spots that may not have been segmented and indexed. Potentially overlapping spots are detected and optionally removed from the grains for better reconstruction of grain shape, or for orientation and strain fitting.

Grain consistency is maintained throughout the indexing process. The maximum number of Friedel pairs that can occur from a given $(h k l)$ plane in a $360^{\circ}$ scan is two. If this is exceeded, only the best fitting two Friedel pairs are kept in the grain, and the remaining pairs are moved into the unindexed set.

The iteration steps can be skipped and the indexing can be used in a single run if the data set constitutes a low risk for erroneous assignments (little strain and mosaicity, accurate segmentation). Steps 4-6 are optional but are expected to improve the grain shape reconstructions. The typical running time on a single processor in iterative mode excluding steps 5 and 6 is of the order of minutes for hundreds of grains and tens of minutes for thousands of grains.

\subsection{Reconstruction}

The three-dimensional shapes of indexed grains are reconstructed individually from their diffraction spots before being assembled in a grain map. Algebraic reconstruction techniques (Kak \& Slaney, 1998) have proved to be successful in reconstructing both convex and concave grain shapes, despite the limited number of available projections (10-100; Fu et al., 2006; Batenburg et al., 2010). A SIRT algorithm from the open-source tomography library ASTRA (Palenstijn et al., 2012) has recently been integrated into the processing route. It enables reconstructions from arbitrary three-dimensional oblique angle projections and thus is well adapted to the diffraction geometry. The library uses GPU acceleration, which results in reconstruction times as short as a few seconds even for large grains. For the best reconstruction quality, the diffraction spots are segmented using soft thresholding (see \$2.1). Their integrated intensities are normalized to unity, which is again physically correct as they represent the same grain with a given volume.

The projection geometry is calculated from the fitted grain orientation (and strain) as the predicted diffracted beam directions. Accounting for the average strain state of the grains is expected to provide improved accuracy as it influences the lattice plane orientations. The projection directions could alternatively be determined from the observed spot centroids and fitted grain centroids. Nevertheless, both are prone to errors and so the approach is expected to be less robust than using the average orientation.

\subsection{Multiphase materials}

DCT has so far been applied to a variety of polycrystalline single-phase materials, including various metals and their 
alloys $(\mathrm{Al}, \mathrm{Mg}, \mathrm{Ti}, \mathrm{Fe}, \mathrm{Ni}, \mathrm{Cu}, \mathrm{U})$, ceramics $\left(\mathrm{Al}_{2} \mathrm{O}_{3}, \mathrm{SrTiO}_{3}\right.$, $\mathrm{UO}_{2}$ ) and some minerals (calcite, basanite, quartz), as well as ice. The extension to dual or multiphase materials is straightforward, provided the individual phases fulfil the basic requirements of DCT in terms of grain size, mosaicity and texture.

Given the crystallographic information (i.e. space group and lattice parameters) for $N$ phases, the list of expected lattice spacings and Bragg angles is calculated. As outlined in \$2.3, Friedel pairs of diffraction spots will be assigned to these phase-specific $\{h k l\}$ families (see Fig. 4). The subsequent processing steps (indexing, searching for individual spots and grain reconstruction) are executed for each phase separately and will result in $N$ three-dimensional phase volumes. If the region occupied by a given phase can be distinguished and segmented from the absorption volume (or from an optional phase contrast reconstruction), the assembly of grains belonging to this phase may be performed under the mask resulting from such a segmentation process.

The different phase volumes are finally merged into a common sample volume in which each voxel is assigned a grain and phase identification number.

\subsection{Combination of data from multiple detectors or scans}

Combining information on the same specimen from different detectors or scans may be desirable to enhance spatial, orientation or strain resolution. To cover a larger solid angle, multiple planar area detectors may be used during a single scan, or consecutive scans if spatial constraints prevent their simultaneous use. When both high spatial and high orientation sensitivity are required, a near-field and a far-field detector may be used together or interchangeably. Synchrotron beamlines often have the capability to support such customized setups, and 3DXRD near-field experiments are often performed at multiple detector distances (Lauridsen $e t$ al., 2001; Suter et al., 2006). An example of a recent dedicated instrument equipped with a semi-transparent high-resolution detector and a second detector at a larger distance from the rotation axis is the one developed in collaboration between Ris $\varnothing$ and the ID11 ESRF beamline (Olsen et al., 2009).

The scattering vectors and/or projections may be combined following the segmentation of diffraction spots either before or after the indexing stage to utilize more reflections for shape reconstruction or strain analysis. When there are enough reflections available, indexing from a single scan is advantageous to avoid introducing additional errors if mechanical drifts occur between the scans. The same argument applies when there is an uncertainty in the relative position of multiple detectors. The fitting procedure in $\$ 2.10$ can be applied using a single or polycrystal standard or the specimen itself to find the absolute or relative positions of multiple detectors. If the data analysis is based on Friedel pairs and more than one detector is used, it is advisable that the setup is chosen in such a way that Friedel pairs can be detected on each individual detector (see \$2.3).

\subsection{Relating two indexed data sets}

Relating two or more indexed data sets of the same sample is a frequent problem where identical grains as well as the global relative displacement and rotation between the data sets are to be found. Such an algorithm has been developed for the DCT software package. Grains are compared on the basis of their centre of mass position, orientation and approximate size in the same way as in the grain merging step of the indexing routine $(\$ 2.5)$ in one of the two sample reference frames. Initial approximate values have to be given for the relative translation and rotation between the data sets. First, grains are matched within preset tolerance limits for the above properties, and the average deviations for successfully matched grains are returned. Orientation deviations are calculated and averaged over the grains as Rodrigues vectors. Other convenient formulations such as quaternions could also be considered to average orientations. The final relative displacement and rotation between the data sets is obtained by applying this procedure iteratively.

The global relative displacement can be computed either from the coordinates of grain centroids or by correlation of voxellated grain maps or alternatively tomographically reconstructed absorption volumes. However, as the sample surface is often smooth, absorption volumes do not usually contain enough contrasting features for this task. The relative rotation can be determined from the difference in orientations, centre of mass positions, correlation of grain maps or a combination of these. A change in the sample environment (mechanical load, temperature etc.) between two scans may change the grain orientations, grain boundaries and even the external surface of the specimen; thus the preferential parameters for calculating the global displacement and rotation should be chosen accordingly. For example, grain growth during annealing results in a change in the grain boundary structure and much less in the orientations, while mechanical loading may affect the mean orientations without a significant change in the grain shapes.

When applicable, grain orientations and centroids provide a faster and potentially more robust method than correlating voxellated grain maps. Finding the relative shifts between data sets on the basis of indexed grains allows for reconstructing the grain maps on the same grid and resampling can be avoided.

An example of a grain map reconstructed separately from two different scans and detector positions is shown in \$3.2.

\subsection{Average elastic strain tensors of the grains}

The average elastic strain state of the grains is inferred from the indexed scattering vectors. Although a far-field setup provides higher angular accuracy owing to reduced susceptibility to motion errors and thermal drifts of the setup (typically much smaller than the pixel size of a diffraction detector), the use of Friedel pairs enables a satisfactory sensitivity for measuring the average elastic strain tensors of grains from near-field diffraction data. Strain accuracy of the order of a few times $10^{-4}$ has been achieved in a DCT 
measurement of a $\beta$-titanium alloy (Reischig, 2008) where the spatial distortion of the detector and the sample drifts during the scan were characterized. The precision of far-field 3DXRD type measurements has been reported to be around $10^{-4}$ (Oddershede et al., 2010; Bernier et al., 2011; Edmiston et al., 2011). The strain calculation described in these references is typically performed by a simultaneous refinement of the grain centre of mass position, orientation and strain (or lattice parameters). These 12 parameters for a grain are found by minimizing the difference between measured and computed diffraction spot positions or scattering vectors.

Here an alternative approach was followed, which can reduce the number of simultaneously fitted parameters to six. The linear formulation described by Reischig (2008) has been replaced with a nonlinear fit that applies no approximation. The input data from the indexed Friedel pairs consist of the diffracted beam paths, the Miller indices, the directions of the plane normals (scattering vectors) as unit vectors and the Bragg angles. The ratio of the theoretical unstrained and measured lattice spacings, the inverse lattice strains, are computed from the Bragg angles. The values are averaged for those $h k l$ reflections for which both Friedel pairs were indexed. In a usual DCT scan the rotational increment is small $\left(0.05-0.1^{\circ}\right)$; therefore not only the Bragg angles but also the orientations of the measured scattering vectors are sensitive to elastic strain in the range of $10^{-3}-10^{-4}$.

Grain positions are defined by a translation and a rotation, i.e. two times three coordinates relative to a known reference crystal, and six strain tensor components with respect to an unstrained reference unit cell. All 12 parameters are defined in the sample reference frame and the transformations are applied in this sequence. The grain position can be fitted as the intersection point of the diffracted beam paths (see $\$ \S 2.3$ and 2.5), independently from the orientation and strain state of the grain. The plane normals are known not in the undeformed state of the crystal but in their deformed state. Consequently, instead of the real strain tensor, an 'unstrain tensor' is fitted, which describes the deformation of the deformed grain into an undeformed crystal in the sample reference frame. For any assumed unstrain tensor, the corresponding plane normal and lattice strain in the undeformed state can be accurately calculated from the measured scattering vectors. The angle between two plane normals calculated in the undeformed state can then be compared with the same interplanar angle in the undeformed reference crystal. Similarly, the measured inverse lattice strain for a given $(h k l)$ plane should correspond to the lattice strain given by the unstrain tensor if the measurement were free of errors. To account for measurement errors the six components of the unstrain tensor are found by minimizing a target function that is the sum of squared deviations of all interplanar angle combinations and inverse lattice strains. The angular and lattice strain components are weighted with the inverse of the corresponding standard deviation. The standard deviations are estimated from the differences measured between the two Friedel pairs of all $h \mathrm{kl}$ reflections for which both pairs were detected. There are at least three noncoplanar plane normals needed to fit all six unstrain components. The strain tensor is calculated from the fitted unstrain tensor in an exact manner. In the case of small deformations, the strain tensor is close to the unstrain tensor multiplied by minus one.

The interplanar angles and lattice strains depend on the deformation (shape and size) of the underlying average crystal unit cell, but none of them are affected by the rotation that defines the orientation of the grain. The three-component Rodrigues vector of the final grain orientation is found by applying this rotation to the unstrained plane normals and minimizing the sum of squared angular deviations from the reference lattice planes in real space.

As an example, the results of the strain and orientation refinement for a titanium sample subject to uniaxial compression are shown in $\$ 3.4$.

\section{Applications}

\subsection{High-resolution DCT grain map of $\mathrm{UO}_{2}$}

An important goal for any $\mathrm{X}$-ray grain mapping technique is to allow smaller grain sizes to be treated, as many real engineering materials have grains sizes of less than $10 \mu \mathrm{m}$. Here we show results from a $\mathrm{UO}_{2}$ ceramic material with a grain size of about $10 \mu \mathrm{m}$. The material is further described by Richard $e t$ al. (2012).

To achieve this spatial resolution, a high-resolution imaging system was used. As for more moderate resolutions, this consisted of a scintillating screen coupled to the ESRF fastreadout low-noise (FReLoN) CCD camera (Labiche et al., 2007) using microscope optics. The light optics give a $50 \times$ total magnification, resulting in an effective pixel size of $300 \mathrm{~nm}$ for the $15 \mu \mathrm{m}$ physical pixel size of the CCD sensor. The system was optimized for high resolution by using a scintillator screen with a very thin active layer $(2 \mu \mathrm{m})$. The thin layer reduces the scintillator point spread and matches the depth of focus $(4 \mu \mathrm{m})$ of the microscope objective used $(20 \times$, numerical aperture 0.45 ). The scintillator material was LSO, which emits light at a wavelength matched to the high-sensitivity E2V chip used in the camera (Martin \& Koch, 2006). An important consideration is that this system is sensitive to light of shorter wavelengths than the original FReLoN camera (550 versus $750 \mathrm{~nm}$ ), giving a proportional improvement in the resolving power of the optical system. A practical consideration is that decreasing the pixel size also reduces the field of view, and hence the required sample-detector distance in order to cover sufficient solid angle to collect diffraction spots. For the highest spatial resolutions and moderate $(\sim 20 \mathrm{keV})$ beam energies it may be necessary to work with the sample only $0.5 \mathrm{~mm}$ from the scintillator, requiring careful sample mounting. At higher energies the diffraction angles are reduced, somewhat relaxing this requirement.

For small grain sizes it is also important to maximize the $\mathrm{X}$-ray flux on the sample, as the intensity in the diffraction spots is proportional to the third power of the grain diameter (assuming kinematical diffraction). This was particularly important for the example discussed here, given the fact that the high density of the sample also required a high beam 
energy $(42 \mathrm{keV})$ to penetrate the sample thickness. At such energies the thin scintillator screens become partially transparent to the X-rays, and the efficiency of conversion of light is reduced. To maximize the flux on the sample the experiment was performed at the ID11 beamline of the ESRF. This has recently been equipped with a cryogenically cooled permanent magnet undulator, giving a flux increase of about a factor of three at this energy. A compound refractive lens transfocator system located close to the primary optics of the beamline was used to condense the beam onto the sample (Snigirev et al., 2009). The resulting scan time was around two hours for 7200 images, giving sufficient diffraction spot intensity for good grain reconstructions.

Some images from the resulting grain map are shown in Fig. 6. The sample consists of a small fragment of $\mathrm{UO}_{2}$ of irregular shape. The maximum sample dimension is about $100 \mu \mathrm{m}$. The DCT process reconstructed 119 grains from the observed diffraction spots. The average grain diameter,

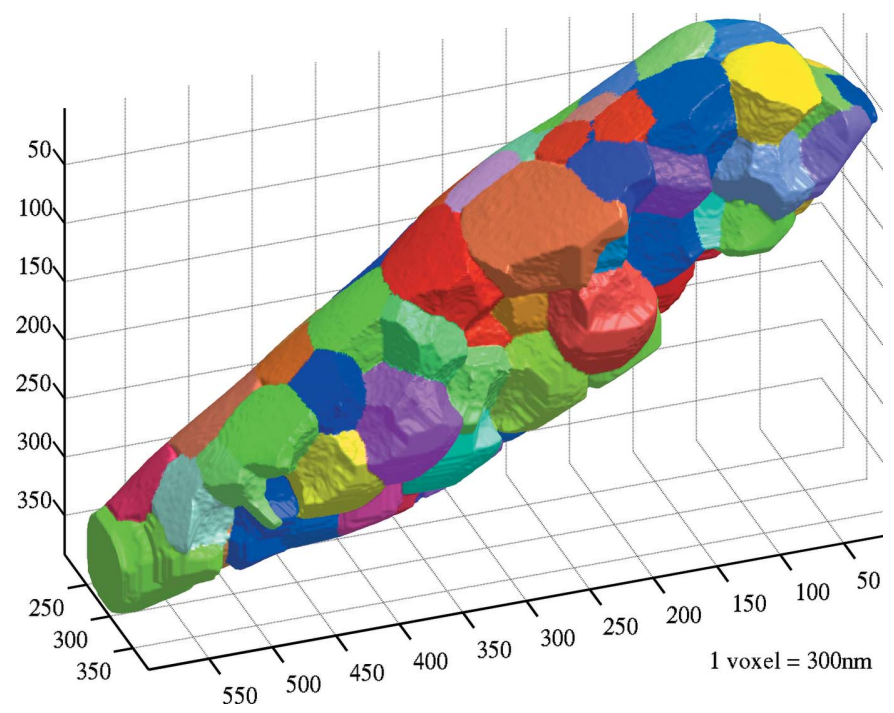

Figure 6

High-resolution DCT grain map of a $\mathrm{UO}_{2}$ sample containing 119 grains. Some grains are not rendered for better visibility of other subsurface grain boundaries.

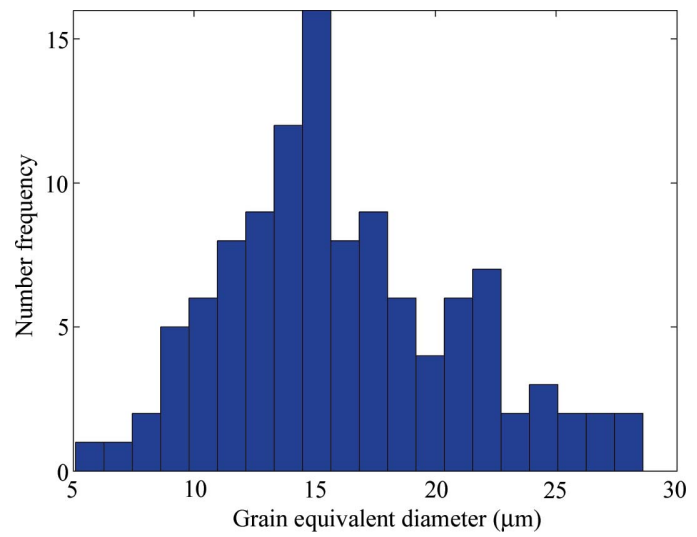

Figure 7

Histogram of the average grain diameters in the $\mathrm{UO}_{2}$ sample shown in Fig. 6. calculated from the grain map, is about $15 \mu \mathrm{m}$. The smallest reconstructed grains are less than $10 \mu \mathrm{m}$ in diameter. Fig. 7 shows a histogram of average grain diameters.

The accuracy of the reconstructed grain shapes is estimated to be around $1 \mu \mathrm{m}$, by considering how well adjacent grains fit together in the final assembled volume before the optional morphological dilation step.

\subsection{Grain mapping and section topography at high Bragg angles}

The reconstruction of grain maps from projections at high Bragg angles and the possibility of simultaneous X-ray topography on sections of selected grains has been demonstrated on a $0.7 \mathrm{~mm}$-diameter sample made of a recrystallized model alloy $(97.5 \% \mathrm{Al}, 2.5 \% \mathrm{Li})$.

The sample was scanned on the ID18F beamline of the ESRF. An undulator source and an $\mathrm{Si}(111)$ double-crystal Bragg monochromator provided a $14.4 \mathrm{keV}$ X-ray beam with a relative energy bandwidth of the order of $10^{-4}$. In addition to the beam-defining slits, an absorbing mask made of gold and with a horizontal gap of $5 \mu \mathrm{m}$ could be translated vertically into the beam to create a line beam profile on the sample. The sample rotation axis was horizontal and perpendicular to the incident beam (Fig. 8).

The setup was equipped with two high-resolution detectors: (A) a FReLoN 2k camera (Labiche et al., 2007) with $3.5 \mu \mathrm{m}$ effective pixel size mounted in the conventional DCT position, centred in the direct beam $4.6 \mathrm{~mm}$ downstream of the sample; (B) a FReLoN E2v camera with $1.5 \mu \mathrm{m}$ effective pixel size mounted $3.65 \mathrm{~mm}$ above the sample, directed downwards. Both detector positions were calibrated on the basis of the Friedel pair data from their own scan, and both sensors contained $2048 \times 2048$ pixels.

DCT scans were performed with each detector over a $360^{\circ}$ continuous rotation in $0.05^{\circ}$ increments. The acquisition time

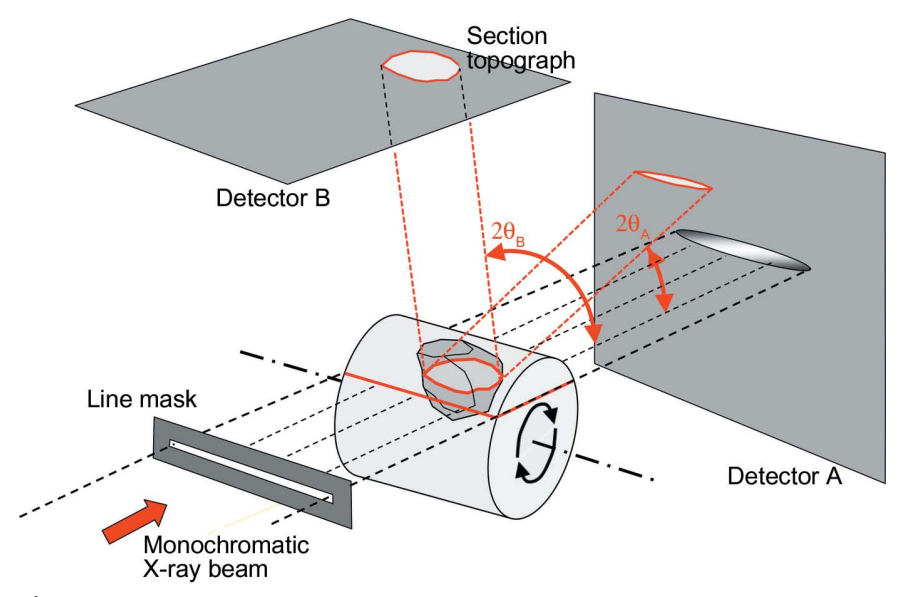

Figure 8

Layout of the setup for combining DCT and section topography with a horizontal rotation axis and vertical detector. An absorption mask provides the line beam illumination on the sample. The irradiated section of the grain is projected almost perpendicularly $\left(2 \theta \simeq 90^{\circ}\right)$ onto the detector plane. An inline detector on which the section appears compressed as it diffracts is also shown for comparison. Grain maps can be acquired by either detector. 
per image was $0.15 \mathrm{~s}$ for detector $\mathrm{A}$ and $0.5 \mathrm{~s}$ for detector B. For these scans the whole sample volume was illuminated.

There were 7290 diffraction spots and 1533 Friedel pairs processed on the forward detector, almost exclusively from the first three $\{h k l\}$ families. The vertical detector covered a $\theta$ range of 15 families, from which 11407 diffraction spots and 2701 pairs were processed. Grains were indexed during the beam time in order to identify interesting grains and reflections for further study.

Following the DCT scans, a grain embedded in the sample was selected. The gold mask was used to create a line beam, and a series of section topographs were recorded with the vertical detector. The line beam was scanned across the grain volume, while integrating over the rotation range of some of its identified reflections.

Corresponding slices from the two grain maps are shown in Fig. 9. The reconstruction from scan A was dilated to create a space-filling map within the mask of its absorption tomogram. As detector B was placed outside the direct beam, no such absorption tomogram was acquired during the scan, and the raw grain map is shown for comparison. In such cases the absorption mask from a detector in the direct beam could be used. All the grains present in A are present in B, but B contains one extra grain at the bottom of the image. The grain shapes agree fairly well in the two reconstructions and differ the most over those areas where the grain sections that fall in the slice are small. The vertical detector in this case provides a better definition map thanks to its smaller pixel size ( $\sim 40 \%$ of the other), so the quality of the maps is not directly comparable.

Two topographs of a Friedel pair recorded close to $2 \theta=90^{\circ}$ at an equivalent height and integrated through $\omega$ show an almost one-to-one projection of the same section (Fig. 10). Deformation inside the grain gives rise to slightly different outlines and different contrast inside the spots. Studying these localized contrast differences in the topographs can give a detailed insight into the lattice deformations at the subgrain level. The combination with the DCT scans allows this information to be extended into three dimensions in a polycrystalline sample.

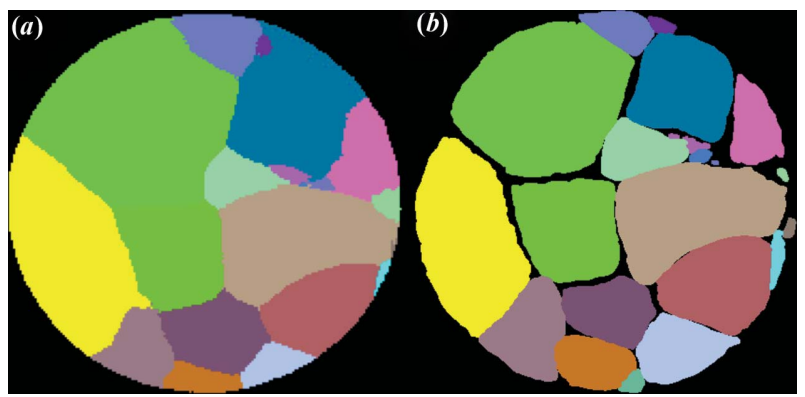

Figure 9

Corresponding slices of reconstructed grain maps of the same $\mathrm{Al}-\mathrm{Li}$ specimen acquired with a horizontal rotation axis and two different detector positions and effective pixel sizes: $(a)$ horizontal detector axis aligned with the incident beam, $3.5 \mu \mathrm{m}$ effective pixel size; $(b)$ vertical detector axis perpendicular to the beam $\left(2 \theta \simeq 90^{\circ}\right), 1.5 \mu \mathrm{m}$ effective pixel size.

\subsection{Multiphase materials: dual-phase steel}

An illustration of a multiphase grain reconstruction is given in Fig. 11. The sample was made from the austenitic-ferritic stainless steel X2CrNiMoN 2253 (1.4462). Accurate DCT reconstructions from this material are challenging for several reasons: the material has a lamellar microstructure and both phases exhibit pronounced texture from the rolling process. Moreover the austenite phase contains about $20-30 \%$ volume fraction of annealing twins. The as-received microstructure was coarsened by heat treatment. Typical intragranular orientation spreads were of the order of $0.2-0.6^{\circ}$.

A total of 3600 images with an effective pixel size of $1.4 \mu \mathrm{m}$ and $2 \mathrm{~s}$ exposure time were collected from the cylindrical sample (diameter $350 \mu \mathrm{m}$ ) positioned $3 \mathrm{~mm}$ from the scintillator (X-ray energy $40 \mathrm{keV}$ ). An additional phase-sensitive tomography scan (600 projections) was recorded at a sampleto-detector distance of $200 \mathrm{~mm}$ but with otherwise identical experiment conditions.

Fig. 11(a) shows a two-dimensional slice of the phase reconstruction using an implementation of the Paganin phase retrieval algorithm (Paganin et al., 2002). This reconstruction shows faint contrast between the regions of austenite (brighter) and ferrite (darker). Figs. 11(b) and 11(c) show the corresponding DCT reconstructions of the austenite and ferrite phase, respectively. A large twin can be seen in the big austenite grain on the right side.

\subsection{Shape and strain information on a large number of grains}

The possibility to obtain combined shape and strain information with a single DCT scan on a large number of grains is demonstrated on a commercially pure titanium sample.

A cylindrical sample was spark cut along the rolling direction (RD) with a diameter of $700 \mu \mathrm{m}$ and scanned on the ID11 beamline of the ESRF. A $40 \mathrm{keV}$ monochromatic X-ray beam with a relative energy bandwidth of the order of $10^{-4}$ was provided by a silicon double-crystal monochromator with its 111 reflection in a horizontally scattering Laue geometry. The rotation axis was horizontal and a detector with an effective pixel size of $1.4 \mu \mathrm{m}$ and a sensor consisting of $2048 \times 2048$ pixels was placed in a regular forward scattering geometry at a

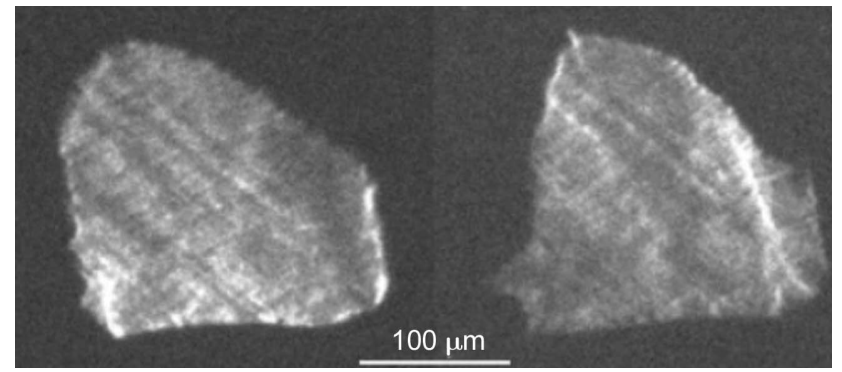

Figure 10

Monochromatic X-ray section topographs of a Friedel pair of a grain embedded in a polycrystalline $\mathrm{Al}-\mathrm{Li}$ sample, acquired with a horizontal rotation and vertical detector $\left(2 \theta \simeq 90^{\circ}\right)$ axis. The images were acquired using the same setup and sample as shown in Fig. $9(b)$. 
distance of $7.6 \mathrm{~mm}$. The field of view allowed the observation of Friedel pairs from the first seven $\{h k l\}$ families with $2 \theta$ angles from 6.96 to $14.27^{\circ}$, with the highest completeness for the first three families: $\{01 \overline{1} 0\},\{0002\},\{01 \overline{1} 1\}$. Images were integrated for $1 \mathrm{~s}$ over a $360^{\circ}$ rotation divided into $0.05^{\circ}$ intervals. In total 106760 diffraction spots were segmented, from which 29296 Friedel pairs were matched and 1755 grains indexed following the geometry calibration.

The first stage of a loading series is presented, with an external compressive load of $15 \mathrm{~N}$ along the sample and rotation axis. The equivalent average normal stress across the cross section equals $39 \mathrm{MPa}$. The deformation due to this load is expected to be in the elastic region, as it is much lower than the yield strength of the bulk material. The load was applied in a compression device that was designed to suit the space constraints of the DCT geometry and allow for irradiation of the sample through $360^{\circ}$.

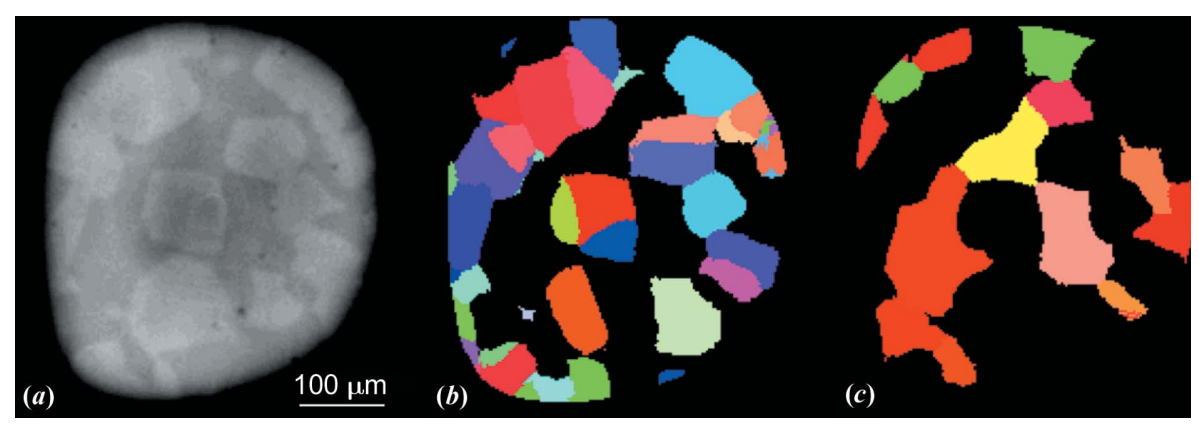

Figure 11

Comparison of phase contrast (PCT) and diffraction contrast tomography (DCT) reconstructions of an austenitic-ferritic dual-phase steel. (a) PCT reconstruction; (b) DCT reconstruction of the austenite phase; (c) DCT reconstruction of the ferrite phase. The PCT reconstruction provides higher accuracy but weak contrast in the grain boundaries. The DCT reconstruction segments the phases and measures grain orientations.
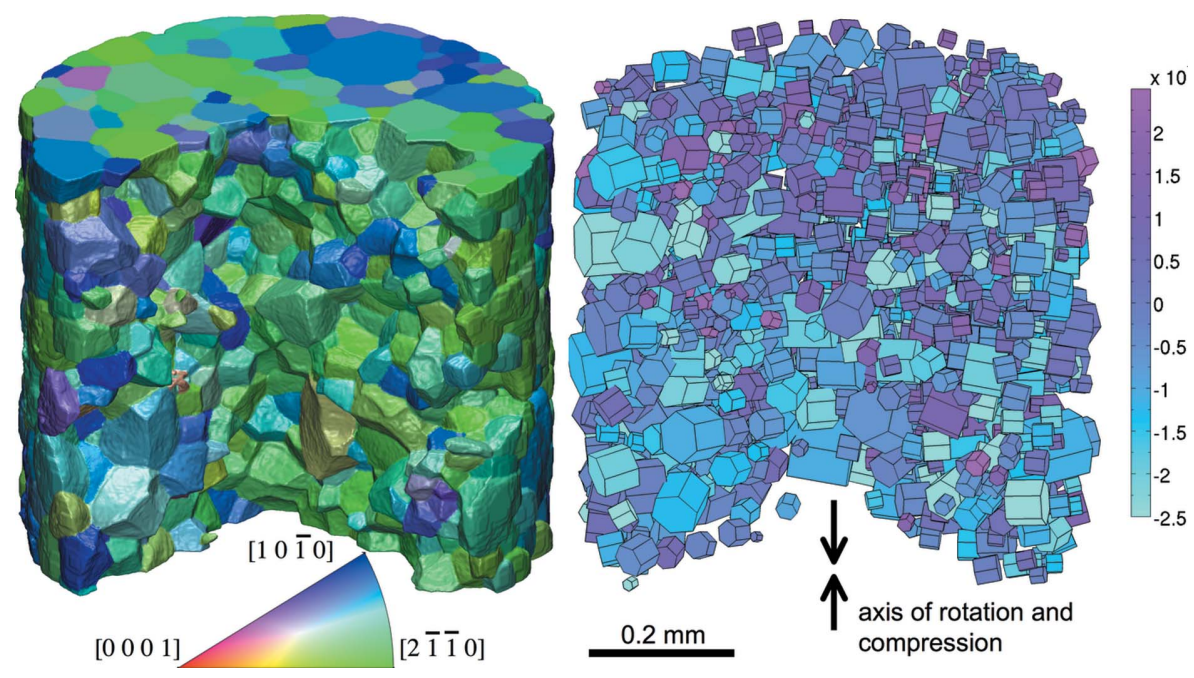

Figure 12

Grain shapes, orientations and elastic strain measured simultaneously in a single DCT scan of a pure Ti specimen. The sample exhibits a strong texture and is loaded in compression. The threedimensional grain map is coloured according to the orientations in the inverse pole figure (left). The same grain locations, orientations and sizes are represented by hexagonal unit cells and colour coded according to the normal strain along the rotation axis and loading direction (right). A quarter of the grains were rendered invisible for better visualization of the internal grain boundaries.
The grain reconstruction and measured strain are presented in Fig. 12, and allow for the observation of texture and strain distribution among the grains. A pole figure of the $\{0002\}$ planes created from the indexed grains is shown in Fig. 13, with a colour scale where random texture would be the equivalent of 1 . Note that the texture seen in this pole figure does not account for small grains that may have been missed in the processing owing to their weak diffraction signal. were computed for each grain. Three comparable effects contribute to the measured strain components: the external load, residual deformation in the material from processing and urement errors. The normal strains along the rotation components: for $85 \%$ of the grains the strains fall in the range between -0.002 and 0.002 . The normal strains in the lateral directions show a narrower distribution, while more than $99 \%$ of the shear strain components fall within this range. The measured average elongation of the grains along the rotation axis is $-4.1 \times$ $10^{-4}$, which can be compared with $-3.5 \times 10^{-4}$, the value calculated from the external load and the average elastic modulus of commercially pure $\mathrm{Ti}$ (110 GPa, approximate value based on manufacturers specifications).

The mean angular deviation between the calculated and measured plane normals (scattering vectors) is $8.60 \times$ $10^{-4} \mathrm{rad}$; its standard deviation is $6.84 \times$ $10^{-4} \mathrm{rad}$. The mean deviation between the computed lattice strains and those measured via the Bragg angles is $11.29 \times$ $10^{-4}$, and its standard deviation is $15.32 \times 10^{-4}$. Note that there was no distortion or sample drift correction applied to these data, both of which may be necessary to improve the strain figures.

\section{Discussion}

\subsection{Setup geometries}

The new DCT analysis approach described in this paper allows an almost arbitrary experimental geometry. This allows scientists to optimize their experiments according to the samples and phenomena to be studied.

Mounting the detector at $90^{\circ}$ to the direct beam exploits the improved sensitivity to elastic strain and misorientation found at higher Bragg angles. At a given energy, strain sensitivity is proportional to $\tan \theta$; thus up to a factor 
of ten may be gained at a perpendicular detector position as opposed to the regular inline geometry. This has been described by Ludwig et al. (2010) for lower-energy X-rays $(\sim 14 \mathrm{keV})$ but could equally be exploited by experiments using cold or thermal neutrons (long wavelengths, giving diffraction angles $2 \theta>90^{\circ}$ ).

As explained by Ludwig et al. (2010) high Bragg angles also enable section topography with optimum spatial resolution. Sections through the sample are illuminated with a pencil or line beam, and near perpendicular projections of the grains intersected are recorded on the detector.

Nevertheless, this geometry has stringent requirements for both the setup and the sample. Synchrotron beams usually have a horizontal polarization, which results in weak elastic scattering in that plane and a strong diffraction signal in the vertical plane. Therefore, the rotation axis used for a DCT scan must be horizontal, which tends to degrade the accuracy of mechanical components owing to the gravitational force acting perpendicular to the rotation axis.

High Bragg angles require lower beam energies for efficient scattering, and hence self-absorption by the sample limits the choice of material and/or sample dimensions. The closer spacing of $\{h k l\}$ families at high $\theta$ angles compensates for the decreased azimuthal range intercepted by the detector and results in a comparable number of diffraction spots in both configurations. While high Bragg angles bring the advantage of reduced extinction (increase of Pendellösung length with increasing $h \mathrm{kl}$ ) and enhanced sensitivity to crystal lattice deformations, higher deformation would eventually challenge the parallel beam reconstruction that is currently used.

At moderate beam energies, intermediate detector positions may be used to optimize the number of reflections on the detector, or the strain, orientation and shape sensitivity of the setup, on the basis of the intensity and multiplicity of the reflecting $\{h k l\}$ families.

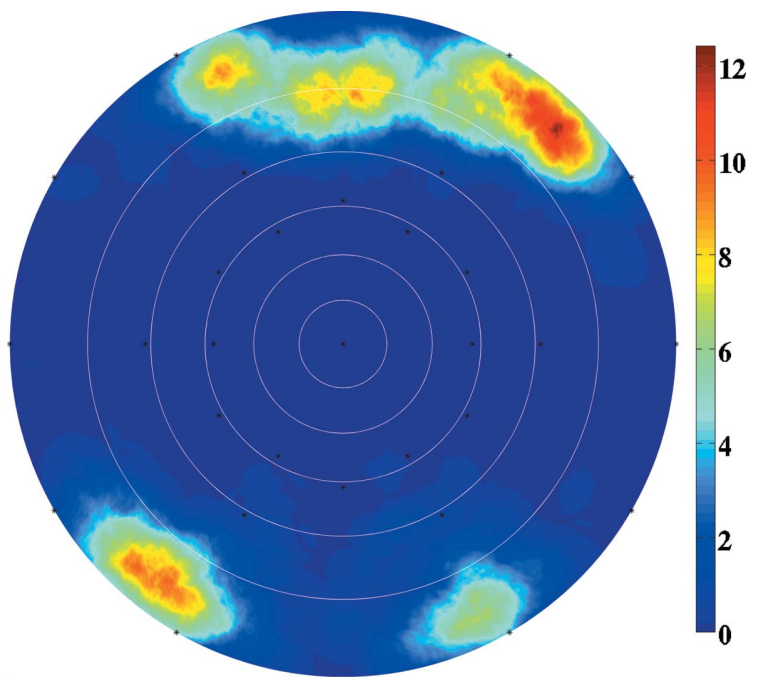

Figure 13

Pole figure of the $\{0002\}$ planes showing the texture of a pure Ti specimen (same as in Fig. 12) as measured by DCT. The normal direction is along the axis of rotation and the cylindrical sample; the intensity is in multiples of the random distribution (m.r.d.).
Placing the detector in near-forward scattering geometry, but offset to one side of the direct beam, could offer advantages in some circumstances, by covering higher $\theta$ angles, and hence more $\{h k l\}$ families, than an inline detector. As an option the direct beam could be captured at the edge of the detector. Roughly half the azimuthal range would be covered, and the total number of observed diffraction spots of the first families would be reduced.

In all the above cases, Friedel pairs can still be observed and exploited for geometrical information, when the sample rotation axis remains normal to the direct beam, and the detector and the sample are in the same plane perpendicular to the axis.

\subsection{Limitations to the grain reconstruction}

In the current DCT approach, diffraction spots are assumed to be parallel projections of the entire grain volume and their integrated intensity is normalized. However, if there are significant orientation and strain gradients inside the grains, the diffraction spots may spread over a wide rotation range and are no longer parallel projections of the grains. In such cases, the reconstruction problem becomes very challenging, as besides the grain shape it should also account for solving the projection geometry as a function of the local orientation and strain. Applying the current reconstruction principles and representing orientation as a three- and strain as a six-valued vector field, ten parameters would need to be found per voxel.

On the other hand, if the crystals are nearly perfect and of large size (several tens of micrometres) then dynamical diffraction effects can occur, introducing intensity variations in the diffraction spots; hence the assumptions of the reconstruction may break down. This is seldom the case with metallic samples but is more likely to occur with semiconductors, ceramics and ice crystals, for example.

Twinning in a material poses a challenge to the current backprojection-based approach in many ways. Small twinned crystals may give diffraction spots that are too weak to be segmented from the background noise of the images, and therefore these spots will not be indexed. It is only when the twins are large enough for the spatial resolution of the setup that they may successfully be indexed and reconstructed as grains separate from the parents. In this case orientation relationships have to be checked during the grain assembly operation in order to allow reconstructed twin grains to be placed inside the parent grains. This contrasts to the normal assembly procedure in which disputed voxels would not be assigned to any grain.

Furthermore, twins share part of their diffraction spots with the parent grain, and their respective contributions to these spots cannot be distinguished. As the entire grain volume is not present in every projection, normalization of the spot intensities to the same value cannot be done in advance.

More accurate reconstruction of twinned microstructures will require a dedicated grain-by-grain reconstruction algorithm where the parent and its potential twins are reconstructed simultaneously assuming all possible twinning orientations. 
Both deformed and twinned microstructures are currently best handled in two dimensions (line beam illumination) by ray-tracing type algorithms (Schmidt et al., 2008; Suter et al., 2006); in this case mosaicity is intrinsically handled in the solution, which is sought as an orientation map rather than individual grains.

Strong texture in a sample increases the chances of spot overlap because grains with similar orientations have a higher probability of diffracting simultaneously in a similar direction. This may present a challenge to the segmentation and indexing procedure even in moderately deformed materials.

\section{Perspectives}

\subsection{Processing software}

DCT has evolved over the past five years to become a grain mapping technique available to users of the ID11 or potentially other beamlines at the ESRF. The current ambition of its authors is that it should be possible for a nonspecialist user to apply for beamtime, record DCT data and reconstruct the results themselves, with limited support from beamline staff. Future developments will focus on further improving the usability of the software, and on better incorporating information from multiple detectors and scanning geometries (e.g. extended beam combined with section topography).

As described in $\$ 4.2$, deformed materials as well as strongly textured and twinned microstructures can cause problems to the current DCT methodology. Subgrain misorientations cause the diffracted beams to deviate from parallel projections. We consider this effect to be the most important limitation on the accuracy of current grain shape reconstructions. Twinned microstructures present a somewhat similar case, in which certain diffraction spots include intensity from both the parent grain orientation and the twin, while others contain only one or the other. In both cases, improving the reconstruction of the grain shapes requires that the spatial distribution of grain orientation is handled in the reconstruction process. Thus, improving the reconstruction of grain shapes and resolving subgrain misorientations are implicitly linked. In previous work we have attempted to reconstruct subgrain misorientation as a function of position within each grain. Initial attempts have been reported by King et al. (2010). Future efforts will focus on developing algorithms based on new concepts in numerical optimization (e.g. compressive sensing), which will refine current DCT maps by adding this level of detail, with the aim of continuing to work in a three-dimensional (extended beam rather than line beam) geometry in order to maintain the advantages that this offers in acquisition time.

\subsection{Improved detectors}

Detector technology continues to improve, and the DCT technique can be expected to evolve in response. The most important aspects are the number of pixels, read-out time and detector optics.

Detectors with greater numbers of pixels will allow smaller effective pixel sizes without reducing the field of view. This will improve the spatial resolution of grain maps, or alternatively allow more grains to be recorded in a single scan. Preliminary tests have been made using a $4 \mathrm{k} \times 4 \mathrm{k}$ FReLoN camera. It is reasonable to think that 10000 grains could be reconstructed from a single scan in a suitable sample using such an imaging system.

Novel detector optics designs and semi-transparent scintillators (Olsen et al., 2009) will make it possible to record diffraction patterns at multiple distances simultaneously by allowing partial transmission of the diffracted beams through the first scintillation layer. Near-field data for grain shape reconstruction and far-field data for enhanced strain sensitivity or more robust indexing could thus be acquired in a single scan and exploited by improved data analysis algorithms.

\subsection{Fast acquisition}

New developments could allow faster data acquisition. The current $2 \mathrm{k} \times 2 \mathrm{k}$ FReLoN camera can be used in frametransfer mode, in which the effective dead time between frames is reduced to $4.2 \mathrm{~ms}$, at the cost of reducing the image size to $2 \mathrm{k} \times 1 \mathrm{k}$ (half of the CCD chip is used as a memory buffer). In this mode, frame rates of more than 30 frames per second can be obtained and a whole data set from a sample containing thousands of grains recorded in a few minutes. Other detector technologies such as CMOS (complementary metal oxide semiconductor) allow even higher frame rates (100s-1000s of frames per seconds full frame) (Rack et al., 2010)

The low efficiency of high-resolution optics means that most grain mapping experiments are flux limited rather than detector readout limited. However, fast detectors offer many interesting possibilities. If the readout time or dead time between images becomes negligible, the rotation stage can be rotated continuously with no significant gap in angular coverage between images. Usually, to completely cover an angular range it is necessary to rewind the rotation stage between frames, or to make a second rotation filling in the gaps between frames. Other types of experiment also become possible. An initial grain map may be made using DCT, taking perhaps one hour. Subsequent grain-resolved dynamic changes in the sample (changes in grain volume, orientation, elastic strain or mosaicity, for example) may be studied using a lower-resolution detector at high speed.

\section{Conclusion}

DCT is a versatile tool for studying single- or multiphase polycrystalline samples and their mechanical or microstructural processes where the grain or grain boundary structure at the micrometre length scale is of interest. The setup allows for a combination of techniques to be used within a single experiment, such as absorption or phase contrast tomography, and line or section topography. The integration of these data gives unprecedented details on the lattice deformation state, which can be extended to three dimensions 
over grains embedded in the bulk of the sample. The flexibility in the experimental geometry offers the possibility to use reflections at high Bragg angles and optimize the detector position for enhanced spatial, strain or orientation sensitivity. Examples of grain maps reconstructed from a high spatial resolution $(\sim 1 \mu \mathrm{m})$ and a high Bragg angle $\left(2 \theta \simeq 90^{\circ}\right)$ setup were shown. Other examples demonstrated the ability to handle multiphase samples, and the possibility to obtain combined shape, orientation and strain information on a large number of grains. The future possibilities and limitations of studying deformed materials and lattice deformations at the subgrain level were discussed.

The authors are grateful for the help of Sébastien Petitdemange and Jon Wright (ESRF) with the experimental hardware and acquisition macros. Special thanks go to Jon for the discussions concerning acquisition procedures and data processing. Furthermore, we thank Stefan Schmiederer (University of Manchester) for his contributions to the DCT software. The $\mathrm{UO}_{2}$ sample and experimental results were obtained through a collaboration with Hervé Palancher (CEA, France) and Anne Bonnin (ESRF). The duplex steel sample was provided by Alexander Giertler (Fachhochschule Osnabrück, Germany). ESRF and its support staff are acknowledged for beamtime on beamlines ID11 and ID18F. KJB was supported by the Netherlands Organization for Scientific Research, research programme 639.072.005.

\section{References}

Alpers, A., Poulsen, H. F., Knudsen, E. \& Herman, G. T. (2006). J. Appl. Cryst. 39, 582-588.

Aydiner, C. C., Bernier, J. V., Clausen, B., Lienert, U., Tomé, C. N. \& Brown, D. W. (2009). Phys. Rev. B, 80, 024113.

Batenburg, K. J., Sijbers, J., Poulsen, H. F. \& Knudsen, E. (2010). J. Appl. Cryst. 43, 1464-1473.

Bernier, J. V., Barton, N. R., Lienert, U. \& Miller, M. P. (2011). J. Strain Anal. Eng. Des. 46, 527-547.

Cloetens, P., Pateyron-Salomè, M., Buffière, J. Y., Peix, G., Baruchel, J., Peyrin, F. \& Schlenker, M. (1997). J. Appl. Phys. 81, 5878-5886.

Edmiston, J. K., Barton, N. R., Bernier, J. V., Johnson, G. C. \& Steigmann, D. J. (2011). J. Appl. Cryst. 44, 299-312.

Fu, X., Knudsen, E., Poulsen, H. F., Herman, G. T., Carvalho, B. M. \& Liao, H. Y. (2006). Opt. Eng. 45, 116501.

Herbig, M., King, A., Reischig, P., Proudhon, H., Lauridsen, E. M., Marrow, J., Buffière, J. \& Ludwig, W. (2011). Acta Mater. 59, 590601.

Johnson, G., King, A., Honnicke, M. G., Marrow, J. \& Ludwig, W. (2008). J. Appl. Cryst. 41, 310-318.

Kak, A. \& Slaney, M. (1998). Principles of Computerized Imaging. New York: IEEE Press.

King, A., Johnson, G., Engelberg, D., Ludwig, W. \& Marrow, J. (2008). Science, 321, 382-385.

King, A., Ludwig, W., Herbig, M., Buffière, J., Khan, A., Stevens, N. \& Marrow, T. (2011). Acta Mater. 59, 6761-6771.

King, A., Reischig, P., Martin, S., Fonseca, J., Preuss, M. \& Ludwig, W. (2010). Proceedings of the 31st Ris $\phi$ International Symposium of Materials Science, pp. 43-57. Roskilde: DTU Press.

Labiche, J., Mathon, O., Pascarelli, S., Newton, M. A., Ferre, G. G., Curfs, C., Vaughan, G., Homs, A. \& Carreiras, D. F. (2007). Rev. Sci. Instrum. 78, 091301.
Larson, B. C., Yang, W., Ice, G. E., Budai, J. D. \& Tischler, J. Z. (2002). Nature, 415, 887-890.

Lauridsen, E. M., Schmidt, S., Suter, R. M. \& Poulsen, H. F. (2001). J. Appl. Cryst. 34, 744-750.

Ludwig, W., King, A., Reischig, P., Herbig, M., Lauridsen, E., Schmidt, S., Proudhon, H., Forest, S., Cloetens, P., Rolland du Roscoat, S., Buffière, J., Marrow, T. \& Poulsen, H. (2009). J. Mater. Sci. Eng. A, 524, 69-76.

Ludwig, W., Reischig, P., King, A., Herbig, M., Lauridsen, E. M., Marrow, T. J. \& Buffière, J. Y. (2009). Rev. Sci. Instrum. 80, 033905.

Ludwig, W., Reischig, P., King, A., Herbig, M., Proudhon, H., Rutishauser, S. \& David, C. (2010). Proceedings of the 31st Ris $\varnothing$ International Symposium on Materials Science, pp. 317-328. Roskilde: DTU Press.

Ludwig, W., Schmidt, S., Lauridsen, E. M. \& Poulsen, H. F. (2008). J. Appl. Cryst. 41, 302-309.

Lyckegaard, A., Alpers, A., Ludwig, W., Fonda, R. W., Margulies, L., Götz, A., Sørensen, H. O., Dey, S. R., Poulsen, H. F. \& Lauridsen, E. M. (2010). Proceedings of the 31st Ris $\varnothing$ International Symposium on Materials Science, pp. 329-366. Roskilde: DTU Press.

Martin, T. \& Koch, A. (2006). J. Synchrotron Rad. 13, 180-194.

Moscicki, M., Kenesei, P., Wright, J., Pinto, H., Lippmann, T., Borbély, A. \& Pyzalla, A. (2009). Mater. Sci. Eng. A, 524, 64-68.

Oddershede, J., Schmidt, S., Poulsen, H. F., Sørensen, H. O., Wright, J. \& Reimers, W. (2010). J. Appl. Cryst. 43, 539-549.

Olsen, U., Schmidt, S., Poulsen, H., Linnros, J., Yun, S., Di Michiel, M. \& Martin, T. (2009). Nucl. Instrum. Methods Phys. Res. Sect. A, 607, 141-144.

Paciorek, W. A., Meyer, M. \& Chapuis, G. (1999). Acta Cryst. A55, 543-557.

Paganin, D., Mayo, S. C., Gureyev, T. E., Miller, P. R. \& Wilkins, S. W. (2002). J. Microsc. 206, 33-40.

Palenstijn, W. J., Batenburg, K. J. \& Sijbers, J. (2011). J. Struct. Biol. 176, 250-253.

Palenstijn, W. J., van Aarle, W., Batenburg, K. J. \& Sijbers, J. (2012). ASTRA. Vision Lab, University of Antwerp, Wilrijk, Belgium, http://visielab.ua.ac.be/software.

Poulsen, H. F. (2004). Three-Dimensional X-ray Diffraction Microscopy. Mapping Polycrystals and Their Dynamics. Berlin: Springer.

Poulsen, H. F. (2012). J. Appl. Cryst. 45, 1084-1097.

Rack, A., Garcia-Moreno, F., Schmitt, C., Betz, O., Cecilia, A., Ershov, A., Rack, T., Banhart, J. \& Zabler, S. (2010). J. X-ray Sci. Technol. 18, 429-441.

Reischig, P. (2008). MSc thesis, Delft University of Technology, The Netherlands.

Richard, A., Palancher, H., Castelier, É., Micha, J.-S., Gamaleri, M., Carlot, G., Rouquette, H., Goudeau, P., Martin, G., Rieutord, F., Piron, J. P. \& Garcia, P. (2012). J. Appl. Cryst. 45, 826-833.

Rolland du Roscoat, S. R., King, A., Philip, A., Reischig, P., Ludwig, W., Flin, F. \& Meyssonnier, J. (2011). Adv. Eng. Mater. 13, 128-135.

Schmidt, S., Olsen, U., Poulsen, H., Sørensen, H., Lauridsen, E., Margulies, L., Maurice, C. \& Juul Jensen, D. (2008). Scr. Mater. 59, 491-494.

Sharma, H., Huizenga, R. M. \& Offerman, S. E. (2012a). J. Appl. Cryst. 45, 693-704.

Sharma, H., Huizenga, R. M. \& Offerman, S. E. (2012b). J. Appl. Cryst. 45, 705-718.

Snigirev, A., Snigireva, I., Vaughan, G., Wright, J., Rossat, M., Bytchkov, A. \& Curfs, C. (2009). J. Phys. Conf. Ser. 186, 012073.

Suter, R. M., Hennessy, D., Xiao, C. \& Lienert, U. (2006). Rev. Sci. Instrum. 77, 123905.

Syha, M., Rheinheimer, W., Bäurer, M., Lauridsen, E., Ludwig, W., Weygand, D. \& Gumbsch, P. (2012). J. Scr. Mater. 66, 1-4.

Tamura, N., MacDowell, A. A., Celestre, R. S., Padmore, H. A., Valek, B., Bravman, J. C., Spolenak, R., Brown, W. L., Marieb, T., Fujimoto, H., Batterman, B. W. \& Patel, J. R. (2002). Appl. Phys. Lett. 80, 3724-3727. 